International Journal of Modern Physics C

(C) World Scientific Publishing Company

\title{
Evolution of population with sexual and asexual reproduction in changing environment
}

\author{
Mingfeng He \\ Department of Applied Mathematics, Dalian University of Technology, \\ Institute of undergraduate innovation, Dalian University of Technology, \\ Dalian 116024, China \\ Hongbo Ruan \\ Department of Civil Engineering, Dalian University of Technology, \\ Institute of undergraduate innovation, Dalian University of Technology, \\ Dalian 116024, China \\ E-mail: ruan_123@tom.com \\ Changliang $\mathrm{Yu}$ \\ Department of Automation, Dalian University of Technology, \\ Institute of undergraduate innovation, Dalian University of Technology, \\ Dalian 116024, China \\ Lei Yao \\ Department of Automation, Dalian University of Technology, \\ Dalian 116024, China \\ Received (received date) \\ Revised (revised date)
}

\begin{abstract}
Using a lattice model based on Monte Carlo simulations, we study the role of the reproduction pattern on the fate of an evolving population. Each individual is under the selection pressure from the environment and random mutations. The habitat ("climate") is changing periodically. Evolutions of populations following two reproduction patterns are compared, asexual and sexual. We show, via Monte Carlo simulations, that sexual reproduction by keeping more diversified populations gives them better chances to adapt themselves to the changing environment. However, in order to obtain a greater chance to mate, the birth rate should be high. In the case of low birth rate and high mutation probability there is a preference for the asexual reproduction.
\end{abstract}

Keywords: Evolution; Sexual; Asexual; Aging.

\section{Introduction}


Most of today's higher species prefer sexual over asexual reproduction, and even asexual species often employ some form of genetic recombination. There are many theories but no consensus ${ }^{1}$ why sex is preferred. The traditional explanation for sex is that it accelerates adaptation by allowing two or more beneficial mutations that have appeared in different individuals to recombine within the same individual ${ }^{2}$. Sex produces variation and thereby promotes evolutionary adaptation. But how does sex achieve this effect, and under what circumstances is it worthwhile?

In the Redfield model, computer simulations have given clear advantages of one or the other way, depending on the parameters. A genomic bit-string model without ageing ${ }^{3,4}$ also justified sexual reproduction; in one case the asexual way of life even died out. Computer simulations based on the Penna model ${ }^{5,6,9}$ have shown that sexual reproduction leads to populations with larger variety of genotypes, although the survival rates for asexual reproduction could be the same ${ }^{9}$. In particular Sa Martins and Moss de Oliveira ${ }^{7}$ have shown that the sexual reproduction gives a better survival chances for a population in the case of a natural disaster.

Most of those models assumed that the environment is constant and the mutations are detrimental, as is customary in ageing theories ${ }^{8}$. However, a question which is often asked by biologists, but cannot be tested in nature is, what is the role of the changes in the environment and of the mutations in the biological evolution. Both are considered to be important factors in determining the fate of an evolving population. To study the role of the reproduction pattern in the fate of an evolving population, it is necessary to investigate the role played by those two factors. The present paper introduces the following assumptions. In the constantly changing environment, we assume that the mutations are blind. The mutations which are helpful to make the population more adaptive to the environment are called beneficial mutations, and those which are negative for the population to adjust to the environment are called deleterious mutations. Below, we present a Monte Carlo study basing on the above assumptions.

The present paper ignores the intermediate reproduction forms ("meiotic parthenogenesis" and "hermaphroditism", which are less wide spread), and compares sexual (now abbreviated by SR) with asexual reproduction (abbreviated by AR), assuming genetic recombination only in the sexual case. We want to investigate the problem of possible advantages of either of the two above mentioned reproduction mechanisms.

The paper is organized as follows. In Sec. 2, we define our model. In Sec. 3 , we present our findings from the simulations. Finally in Sec. 4, we state our conclusions.

\section{The model}

Consider a square lattice $L \times L$ with periodic boundary conditions, where initially $N(t=0)$ individuals are located. Each of them is characterized by its location $(k)$ at a lattice cell, genotype $\left(g_{k}\right)$, phenotype $\left(h_{k}\right)$ and age $\left(A_{k}\right)$. On each lattice 
cell there might be at most one individual. The space (habitat) as well as time is discrete.

The asexual version (haploid organisms) of the model considers a single string of 32 sites (loci) associated to each individual of a population, representing its genotype. For the sexual case (diploid organisms), each individual has two such strings (chromosomes) read in parallel. Each locus can be in two states, called alleles, and denoted here as 0 and 1 . Thus a genotype is a single or double string of zeros and ones. They are defined at birth, kept unchanged during the individual lifetime.

From a genotype a phenotype is constructed, as a single string of the same length, according to the following rule. For haploid organisms the phenotype is identical with the genotype. The diploid phenotype is constructed as follows - All the loci are sorted as recessive and dominant loci, each of which takes up $50 \%$ of the total loci. On the dominant loci, if a "1" appears (dominant homozygote $(1,1)$ and heterozygote- $(0,1)$ or $(1,0))$, a 1 is put at the corresponding place of the phenotype, otherwise a 0 . While on the recessive loci, if a "0" appears (recessive homozygote- $(0,0)$ and heterozygote- $(0,1)$ or $(1,0))$, a 0 is put at the corresponding place of the phenotype, otherwise a 1 . We interpret the bit-string not only the phenotype but also 32 intervals ("years") in the life of an individual. At a certain age we see only the loci corresponding to this and earlier ages. Age $A_{k}$ is augmented for each individual after completion of one Monte Carlo step (MCS).

The environment is represented in the model by a certain "ideal" phenotype, $H$, which ensures the best adaptation for an individual to the existing conditions. Such "ideal" phenotype has been already introduced in the literature ${ }^{10,11,12}$. The fitness of an individual to the environment is measured as the agreement of the individual's phenotype with the "ideal" phenotype. Thus, there are no absolutely good or bad genes in our model. Only when a locus on the phenotype is identical with the corresponding locus on the "ideal" phenotype, it is considered to be a good one and vice versa. The environment is constantly changing. Therefore the "good" or "bad" for genes are also temporal.

Here we consider random mutations affecting genotypes of the offspring. On each locus of the genotype, a mutation may take place with probability $M$.

The mutation occurs at a randomly chosen bit of the genotype of the offspring, with the probability $M$.

Here we use separate computer simulations for sexual and asexual reproduction. The process starts with an initial population, $N(0)$, having random genotypes $g_{k}$, chosen from a uniform distribution. The dynamical rule is given by the following steps.

(1). An individual $k$ is randomly chosen. In the case of SR, only females are selected.

(2). One search is done for an empty cell in the nearest neighborhood. If not found, the individual stays in place. Otherwise the individual moves to the new position. 
(3). In the case of $\mathrm{SR}$, if the age of the female is between $A_{m}$ and $A_{d}$, a male with age larger than $A_{m}$ is chosen in the Moore neighborhood of the female. If it is found, the two give birth to $q$ offspring, each of which is randomly either male or female. For each of the offspring an independent search is made for a free cell in the Moore neighborhood of the female. If found, the progeny is put there. If the cell is already occupied, nothing happens and we search for a place for the next offspring.

In AR no mate is needed and if the age of the individual is between $A_{m}$ and $A_{d}$, it gives birth to $q$ offspring. The offspring are located on empty cells in the Moore neighborhood of the parent by the same way as the SR.

The age of a newborn baby is set to 1 .

(4). Each offspring receives independently its genotype. The way it is created depends on the kind of population we are considering. For AR without recombination the baby inherits the genotype of its parent, change only by mutations. In the case of SR each offspring receives its own genotype constructed through simple recombination and one gamete coming from each parent. The process goes as follows. The genotype of a parent is cut at a random place. Since the genotype is a double string there are four pieces which are glued across, forming two gametes. Of these one is chosen randomly. The same is done for the second parent. This makes the genotype of the offspring. Then the mutation procedure is applied.

Mutations are realized in the following way. On each locus of the genotype a mutation may take place with probability $M$. The mutation is realized by changing a 1 into 0 , or a 0 into 1 .

Phenotypes of the offspring are constructed from the genotypes according to the rule given above.

(5). The individual $k^{\prime} s$ fitness $p_{k}$, i.e., the degree of agreement of its phenotype, $h_{k}$ with the "ideal" one, $H$, is calculated as the number of bit positions up to $A_{k}$ where phenotype and ideal phenotype agree, divided by the current age $A_{k}$.

The fitness $p_{k}$ is compared with a threshold $D$, when it is lower than this limit, the individual dies.

(6). If the age of the individual $k$ is more than 32 , the individual dies.

(7). After making as many picks as there are in the population at that time, one Monte Carlo Step has been completed. The age of every individual is increased by one.

The changes of the environment are realized as modifications of the "ideal" phenotype, i.e. by substituting a 1 by a 0 in a randomly chosen site (locus), or a 0 by 1 , every $T$ MCS.

The parameters of the model are the following: Linear size of the lattice $L$, the concentration of the initial population $c(0)=N(0) /(L \times L)$, the minimum age $A_{m}$ and the maximum age $A_{d}$ for reproduction, the maximum number $q$ of offspring (birth rate), the probability of a mutation per gene for the babies $M$, the minimum of fitness $D$, the period of changes in the environment $T$.

Since it would be difficult to operate with so many parameters we decided on giving to some of them constant values. We took $A_{m}=8, A_{d}=12, D=0.8, c(0)=$ 
0.8. Simulations were performed on a square lattice of size $L \times L=500 \times 500$. The remaining three parameters - the birth rate $q$, the mutation probability $M$ and the period of changes in the environment $T$, have a more qualitative character.

\section{Results and discussion}

Let us begin by considering the simplest case-the environment constant in time. The changes in the environment are realized here as changes in the "ideal" phenotype with a period $T$, so the $T$ can be taken as an infinite number here. As shown in Fig. 1, with parameters $q=2, M=0.01$, both populations adjust to the environment and reach a high level of concentration. Each population passes through a deep minimum, which also appears in Figs. 2, 3 and 4. This is a crucial moment where the population must overcome the danger of extinction because of stochastic fluctuations. Recovery is faster for AR, which exhibit its advantages of efficient and straightforward reproduction (need no partner to mate) under this condition. The concentration of the equilibrated population is 0.88 in the asexual and only 0.81 in the sexual case. The results are similar to those obtained earlier in the Penna model.

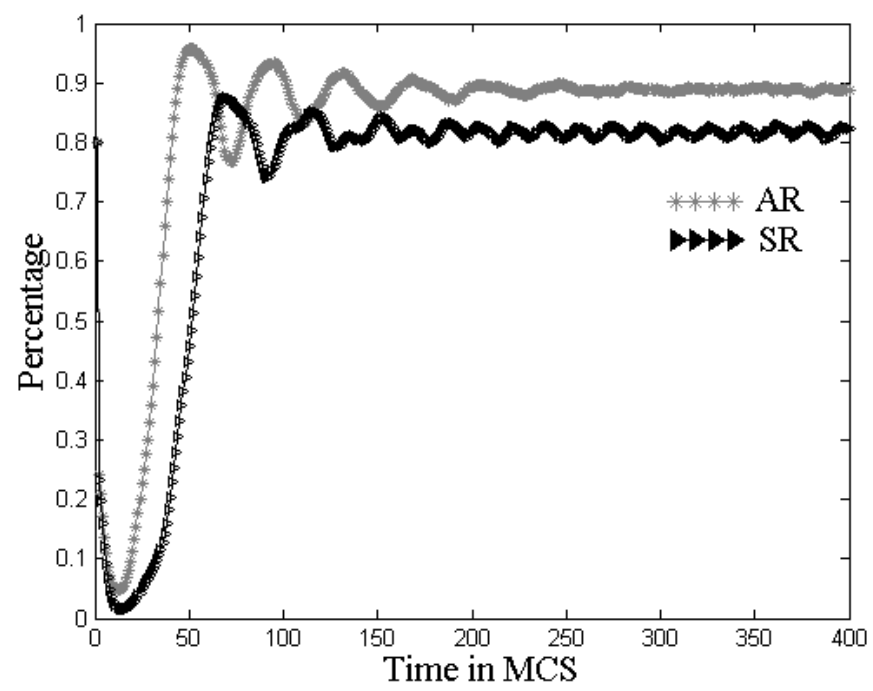

Fig. 1. Concentration versus time (in MCS). $q=2, M=0.01$.

Two reproduction patterns: SR (black triangle) and AR (grey star).

When the period of environmental changes is comparable with the average age of the generation, the effect of environment changes is pronounced. For $T=32 \mathrm{MCS}$, using $q=2$, and $M=0.01$, we have tested that the AR and SR populations may become extinct frequently, see Fig. 2. With very low birth rate and mutation probability, both populations cannot adapt well to the changing environment.

The concentrations of the two populations versus time is shown in Fig. 3(a) for 


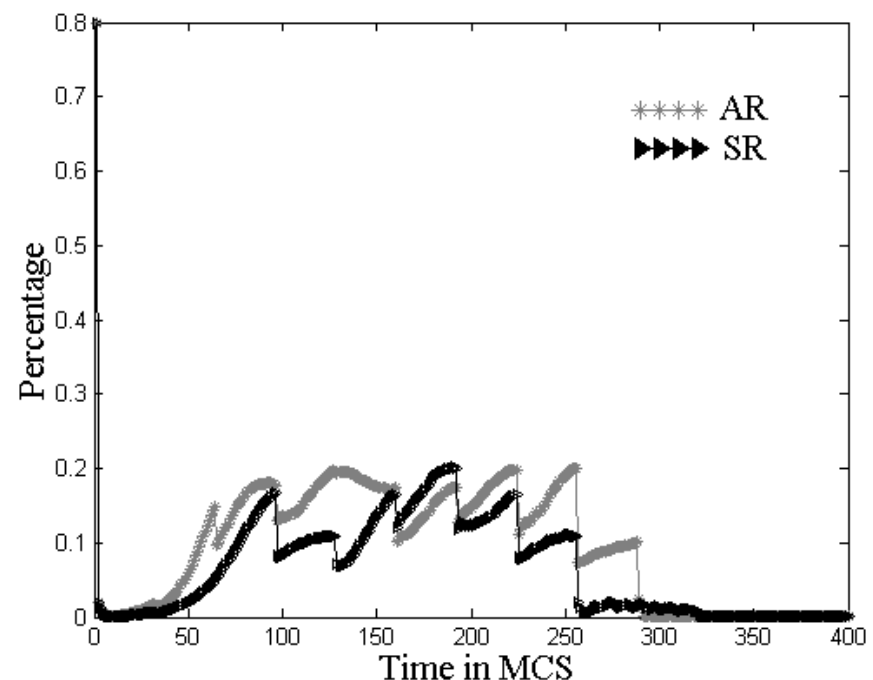

Fig. 2. Concentration versus time (in MCS) $T=32, q=2, M=0.01$. Two reproduction patterns: SR (black triangle) and AR (grey star).

$T=8 \mathrm{MCS}$, and in Fig. 3(b) for $T=32 \mathrm{MCS}$, both keeping $q=2$ and increasing $M$ from 0.01 to 0.15 . For $T=8 \mathrm{MCS}$ (Fig. 3(a)), although the environment is subject to rapid changes, the population of $\mathrm{AR}$ adapts to the environmental changes and its concentration fluctuates around a level of 0.41 . It should be however mentioned that the increase of the mutation probability does not help the SR from extinction. When $T$ is increased to 32MCS (Fig. 3(b)), after surviving the initial sudden drop, concentrations of the two populations (AR and SR) are mounting with time towards higher values. For SR, it fluctuates at a concentration below that of AR. One may conclude that when the birth rate remains at a low level, increasing $M$ is clearly more favorable to the development of the population for AR.

The situation changes drastically, in favor of SR, if the birth rate becomes higher, while the mutation probability remains low. The effect is presented in Figs. 4(a) and (b). Increase $q$ from 2 to 7 , and keep $M=0.01$, then the situation is reversed comparing to Figs. 3(a) and (b) respectively: the SR is doing better than AR for both $T=8$ and 32 . The benefits of SR are enhanced by higher birth rate. It can be interpreted as that the effect of recombination becomes pronounced. But in order to complete recombination, the individual of SR have to find a partner to mate, which is demanding. Thus, only the high birth rate, which may result in a large population, can provide the SR with a greater chance to mate. In the previous models, the partners for mating were selected randomly from the whole population. However, the difficulty of mating for SR, i.e. the spatial limitation, was not considered.

As could be expected, when both the birth rate and the mutation probability 

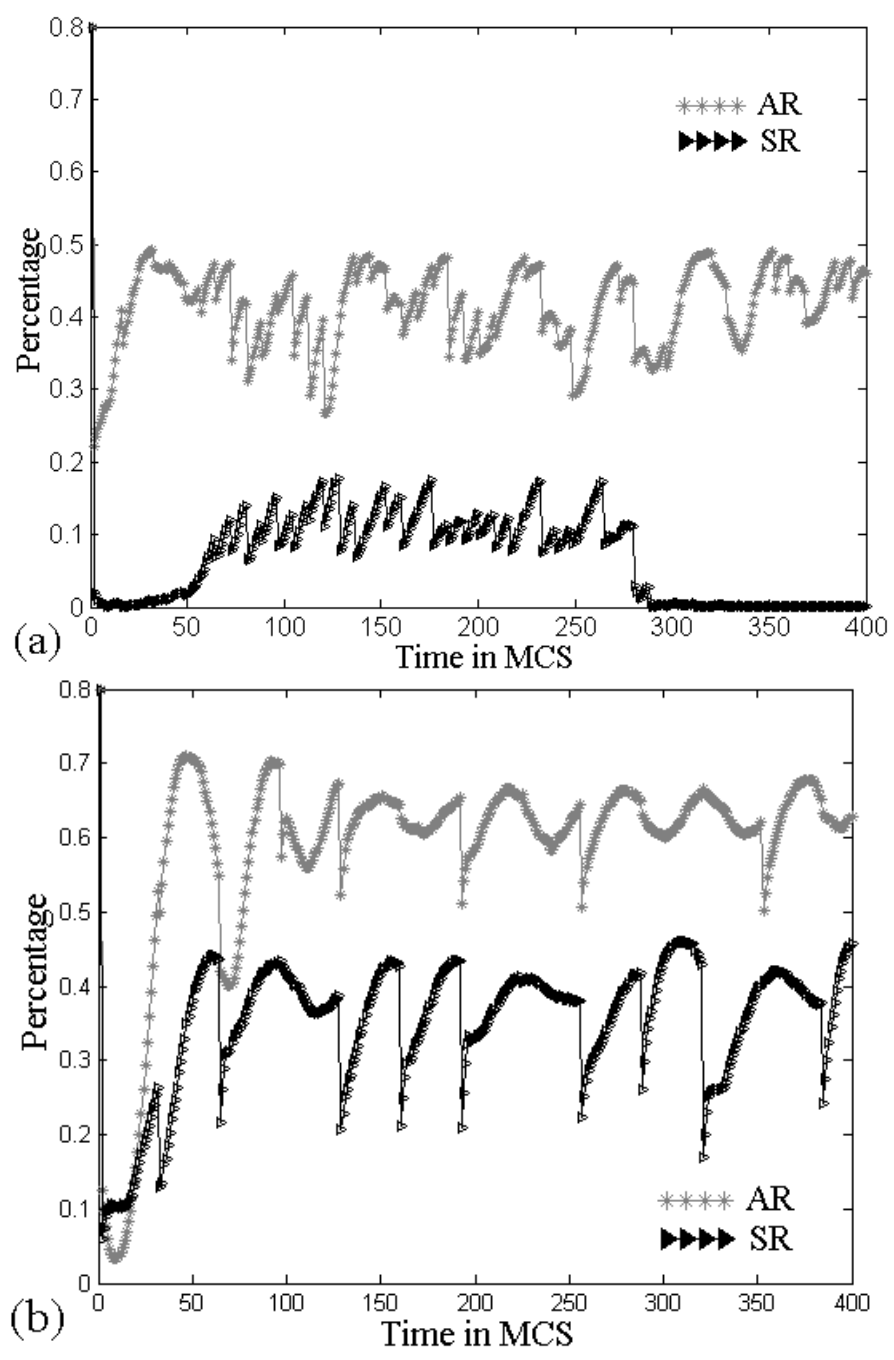

Fig. 3. Concentration versus time (in MCS).

(a) $q=2, M=0.15, T=8$. (b) $q=2, M=0.15, T=32$.

Two reproduction patterns: SR (black triangle) and AR (grey star). 

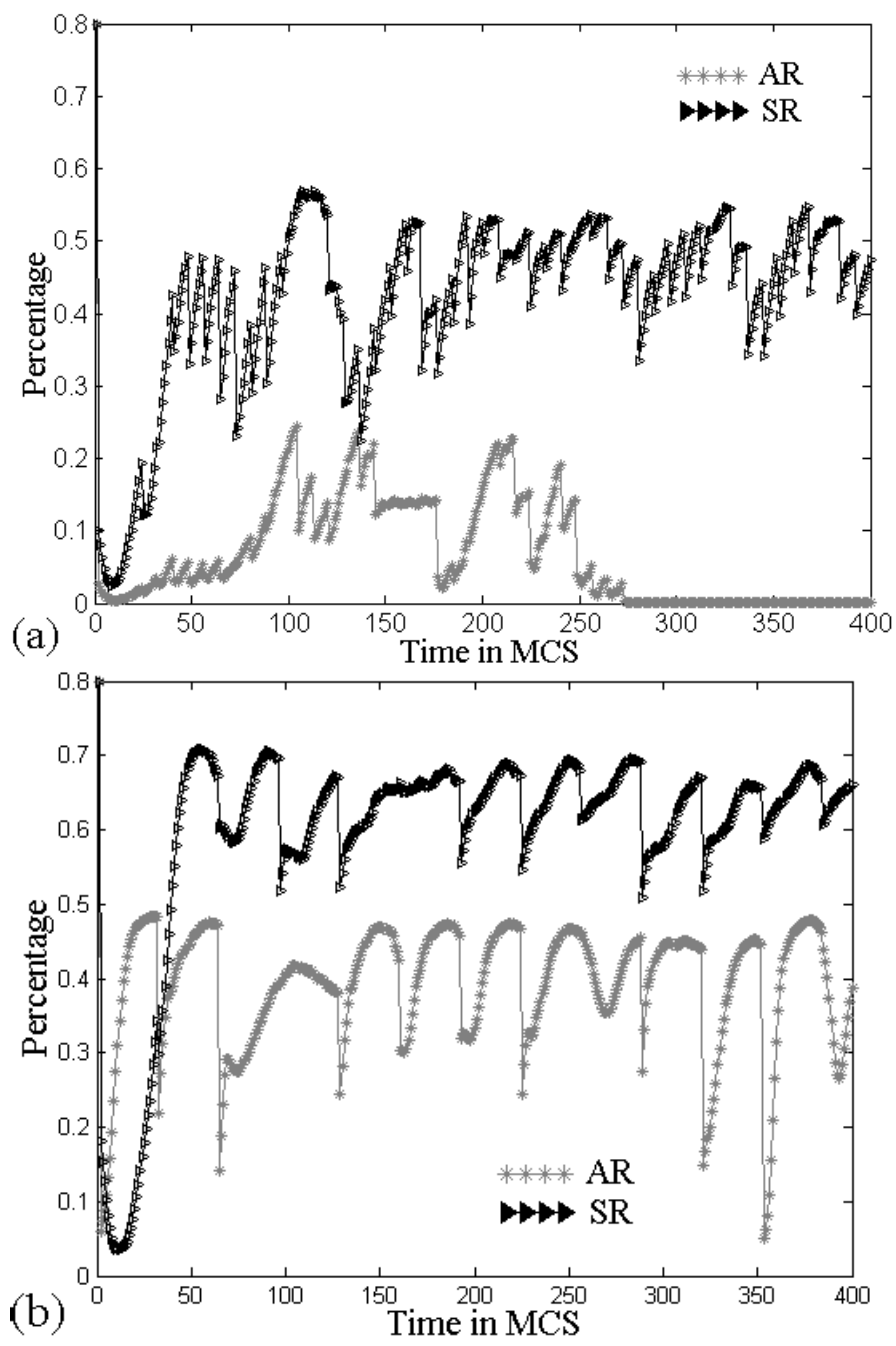

Fig. 4. Concentration versus time (in MCS).

(a) $q=7, M=0.01, T=8$. (b) $q=7, M=0.01, T=32 .$.

Two reproduction patterns: SR (black triangle) and AR (grey star). 
are high, for $T=8 \mathrm{MCS}$ and $32 \mathrm{MCS}$, the populations for AR and SR adjust easily to the changes of the environment and there is a little preference for AR (the figures are not shown here).

For the evolution of a population the vital question is its chances of survival in a given environment. Table 1 shows the percentage of surviving populations after 1000 MCS in a series of 100 runs. As seen from the Table 1, populations with higher mutation probability and birth rate become extinct less frequently. For populations with high mutation probability and low birth rate the AR is the more efficient. When the mutation probability is low and the birth rate is high, the SR is the preferred one.

Table 1. Percentage of surviving populations after 1000 MCS in a series of 100 runs

\begin{tabular}{ccccc}
\hline & $\mathrm{T}=8$ & $\mathrm{~T}=8$ & $\mathrm{~T}=32$ & $\mathrm{~T}=32$ \\
\hline & AR & SR & AR & $\mathrm{SR}$ \\
\hline $\mathrm{q}=2, \mathrm{M}=0.01$ & 0.05 & 0.04 & 0.08 & 0.09 \\
$\mathrm{q}=2, \mathrm{M}=0.15$ & 0.71 & 0.45 & 0.82 & 0.56 \\
$\mathrm{q}=7, \mathrm{M}=0.01$ & 0.48 & 0.80 & 0.54 & 0.84 \\
$\mathrm{q}=7, \mathrm{M}=0.15$ & 0.97 & 0.93 & 0.99 & 0.94 \\
\hline
\end{tabular}

Thus, depending on parameters, either AR or SR can be the clearly preferred choice, in agreement with reality. It is well known that the advantages of AR are efficient and straightforward, here we do not discuss it further and concentrate on the SR.

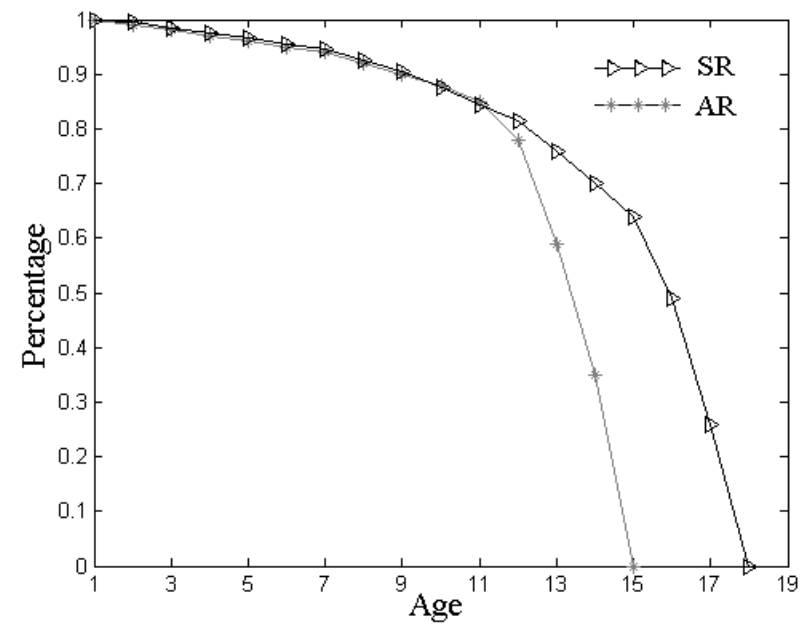

Fig. 5. Age distribution (in percentage) of the AR and SR populations versus age dependence. Parameters: $q=7, M=0.01$ and $T=8$.

Two reproduction patterns: SR (black triangle) and AR (grey star).

For $q=7, M=0.01$ and $T=8$, we present the age structure of populations for 
AR and SR in the steady state, see Fig. 5. The y-coordinate is the ratio between the number of the individuals with a certain age and the number of the whole population. The averaging was done over 50 years.

In case of the AR we can see that the curve goes down slowly, and then, after the maximum reproductive age there is a sudden drop, which has been also mentioned in ${ }^{13,14}$. This phenomenon can be explained as follows. Although the environment is changing, most of the genes on the phenotype which do not adapt to the environment are able to accumulate beyond the maximum reproduction age in time. As to the SR case, the drop is delayed about 3 years and the drop is less steep. Sexual reproduction also ensures that the slowly decreased stage before the drop is maintained longer.

Sexually reproducing individuals, males and females, live longer than the asexually reproducing females. Why can the females survive after their reproductive age is passed in the case of SR while they cannot in the AR case? Since the sex is not transmitted genetically, we take each child as male or female with probability 0.5 . Then either both males and females die soon, or both males and females die late. Thus, males help the survival of younger females beyond their reproduction age.

The above conclusion has some resemblance to the ones obtained in ${ }^{9}$. The difference is that here the curve decreases before the sudden drop because of the pressure of environmental changes, while in ${ }^{9}$ there was hardly any decrease.

\section{Conclusions}

The present paper first introduces the following assumptions. In the constantly changing environment, we assume that the mutations are blind. The mutations which are helpful to make the population more adaptive to the environment are called beneficial mutations, and those which are negative for the population to adjust to the environment are called deleterious mutations.

Basing on the above assumptions, we presented a lattice model of population dynamics based on Monte Carlo simulations. Each individual is characterized, apart from its location on the lattice, by its genotype, phenotype and age. The agreement of the phenotype with the "ideal" phenotype characterizing the environment determines the individual fitness. There are three major factors influencing the evolving process-birth rate, mutation probability and the period of changes in the environment. Depending on the three parameters, either AR or SR can be favorable.

With very low birth rate and mutation probability, both populations cannot adapt well to the changing environment. The AR and SR populations may become extinct frequently. If the mutation probability is high and the birth rate remains at a low level, the advantage of $\mathrm{AR}$ on the adaptation to the changing environment is more pronounced, which is ascribed to its efficient and straightforward nature (need no partner to mate) reproduction scheme. And this preference of the AR disappears if the birth rate of the population is high and the mutation probability remains at a low level. Then the SR is the more favorable, in agreement with the 
conclusion of ${ }^{15}$. The present work supports the claims that SR by keeping more diversified populations gives them better chance to survive cataclysms. However, in order to obtain a greater chance to mate, the birth rate should be high.

In our simulations the populations with different reproduction schemes were not evolving together, hence there was no effect of direct competition. However both evolution processes started from the same conditions, like initial concentration of individuals, distribution of the genotypes and habitat requirements.

\section{References}

1. A.S. Kondrashov, Nature 369, 99 (1994).

2. H.J. Muller, Am., Nature 66, 118 (1932).

3. B. Orcal, E. Tuzel, V. Sevim, N. Jan, A. Erzan, Int. J. Mod. Phys. C 11, 9736 (2000).

4. E. Tuzel, V. Sevim, A.Erzan, eprint cond-mat/0101426

5. S. Moss de Oliveira, P.M.C. de Oliveira, D. Stauffer, Evolution, Money, War and Computers ,(Teubner, Leipzig, 1999).

6. T.J.P. Penna, J. Stat. Phys. 78,1629 (1995).

7. J.S. Sa Martins, S. Moss de Oliveira, Int. J. Mod. Phys. C 9, 421 (1998).

8. K.W. Wachter, C.E. Finch, Between Zeus and the Salmon, The Biodemography of Longevity , (National Academy Press, Washington, DC, 1997).

9. D. Stauffer, P.M.C. de Oliveira, S. Moss de Oliveira, T.J.P. Penna and J.S. Sa Martins, arXiv:cond-mat/0011524 v1 (2000)

10. I. Mroz, A. Pekalski, K. Sznajd-Weron, Phys. Rev. Lett. 76, 3025 (1996).

11. A. Fraser, D. Burnell, Computer Models in Genetics, (McGraw-Hill, New York, 1970).

12. D. Charlesworth, M.T. Morgan, B. Charlesworth, Genet. Res. (Cambridge) 59, 49 (1992).

13. T.J.P. Penna, S. Moss de Oliveira and D. Stauffer, Phys.Rev. E 52, 3309 (1995).

14. T.J.P. Penna and S. Moss de Oliveira, J. Physique I 5, 1697 (1995).

15. A. Pekalski, Eur. Phys. J. B 13, 791 (2000). 